\title{
Active control of transitional channel flows with pulsed and synthetic jets using vortex methods
}

\author{
Emmanuel Creusé ${ }^{1}$, André Giovannini ${ }^{2}$, and Iraj Mortazavi ${ }^{3}$ \\ 1 LAMAV \& INRIA Lille Nord Europe, Université de Valenciennes, 59313 \\ Valenciennes Cedex 09, France. ecreuse@univ-valenciennes.fr \\ 2 IMFT UMR CNRS, 118, route de Narbonne, 31062 Toulouse, France. \\ giova@imft.fr \\ 3 IMB \& INRIA Université Bordeaux I, 351, cours de la Libération, 33405 \\ Talence, France. Iraj.Mortazavi@math.u-bordeaux1.fr
}

Keywords : Vortex method, closed and open-loop active control, backwardfacing step.

\begin{abstract}
In this work a vortex method is used to simulate and control an incompressible two-dimensional transitional flow over a backward-facing step. Two different control strategies are implemented to modify the shedding, the recirculation zone behind the step and the transport in the channel. The first technique consists in using a pulsing inlet velocity and the second one is based on local oscillating jets implemented on the step vertical wall. The influence of these controls on the flow behavior is carefuly investigated. Both, open-loop and closed-loop active control approaches are performed.
\end{abstract}

\section{Context and aim of the study}

The aim of the present work is to develop two different active control strategies to manipulate the flow characteristics inside a backward-facing step channel with a transitional flow regime: 1) using pulsed inlet velocities, with open and closed-loop frequency choices; 2) implementing two jets to the vertical step wall with open-loop or closed-loop action into the lower and upper levels of the step. This work follows the primary results obtained by the authors in a previous paper for laminar flows [3]. Here, the impact of the control on several flow characteristics like vorticity, energy, fluctuations, velocity gradients etc. is carefully analysed. Reynolds numbers are $R e=500$ and $R e=2000$. 


\section{Numerical scheme}

The numerical simulations are performed using a Vortex-In-Cell method [2]. In VIC calculations, an Eulerian grid is implemented in order to compute efficiently the velocity field on the Lagrangian particles. The goal is to obtain a fast computation of the particle velocities in regular bounded domains. Here, fast Poisson solvers enable fast velocity evaluations. Compared with pure finite-difference methods, VIC methods offer the advantage of a robust and accurate treatment of the convective part of equations with time steps not constrained by convective CFL conditions [1]. The simulations are validated, comparing them to existing experimental and numerical literature and grid convergence analysis ensured. Appropriate sets of space and time numerical parameters are selected in order to achieve accurate control strategies [4].

\section{Control strategies}

The control is performed either by taking an oscillating inlet velocity $\mathbf{u}(t)=$ $\left(u_{i n}(t), 0\right)^{T}$, instead of the uncontrolled uniform velocity boundary condition simulation (Fig. 1, Control $_{1}$ ), or by two small jets implemented on the upper and lower parts of the step (Fig. 1, Control $_{2}$ ). Both, open and closed-loop controls are used and compared. The open loop studies are performed for a wide range of parameters to get the most efficient ones. These values are then compared to the closed-loop control to verify its efficiency.

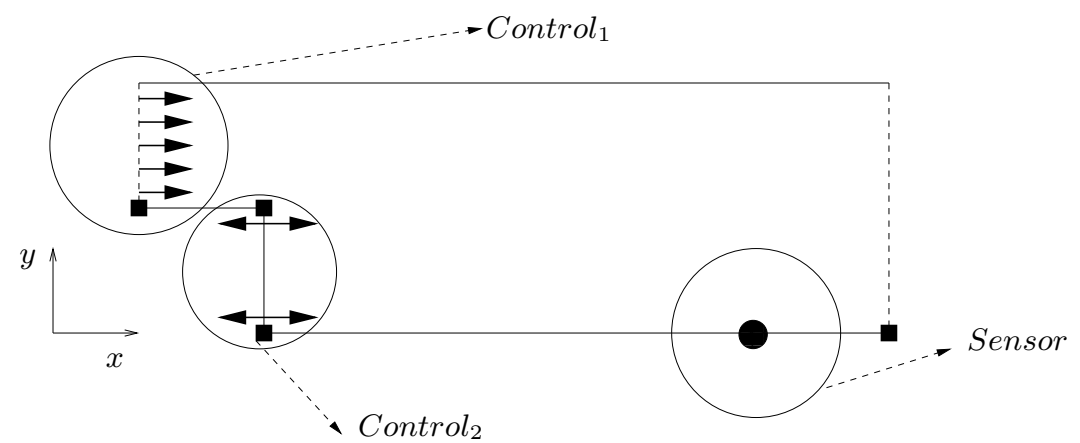

Fig. 1. Control devices on the step geometry.

The control effect is focused on the vortex shedding, transport phenomena and the step recirculation zone behavior. One of the main targets is to reduce the recirculation area and length, then to decrease the trapped particles and to improve the transport of vortices [5]. In order to quantify this control, several time dependent fonctionals are studied [4]. These functionals are the recirculation area length $L_{r}(t)$, the total enstrophy in the domain that measures the 
vorticity of the flowfield and the near wall recirculation area intensity that corresponds to the level of shear forces.

\section{Numerical results}

\subsection{First control type}

The first control (Fig. 1, Control $_{1}$ ) is achieved oscillating the inlet velocity profile $u_{i n}(t)=1.0+A \cos (2 \pi f t)$. The frequency $f$ and the amplitude $A$ of this oscillating flow are obtained either by an extensive parametric study (open-loop control $O L C$ ) or implementing the dominant frequencies of the vorticity formation and transport in the pulsing flow as the reference value (closed-loop control $C L C$ ).

For the open-loop control an extremum value for each time-averaged functional for a frequency nearly equal to the basic fundamental frequency of the flow, namely $f \approx 0.14$ is achieved (see Fig. 2). On the other hand, the efficiency of the control almost linearly increases with the increasing values of the amplitude until a "plateau".

The closed-loop control consists in using the fundamental frequencies of flow shedding or transport in order to fit the oscillation frequency. The frequency measuring sensors may be implemented on the wall (non-intrusive sensors) or inside the flow field (intrusive sensors). The main advantage of the closed-loop control is that the control frequency is taken directly from the principal flow frequencies and avoids the heuristic trial and error approaches with high computational cost needed for open-loop methods. In the non-intrusive closed-loop control the quantity $\partial^{2} u / \partial y^{2}$ is connected to the wall tangent pressure gradient. The closed-loop control consists in taking $u_{i n}(t)=1.0+M\left(\partial^{2} u / \partial y^{2}(t)\right)_{S_{n}}$, where $S_{n}$ is a point on the bottom wall (see the location of the sensor on Fig. 1), and $M=2.5 \cdot 10^{-3}$ is chosen to make vary the inlet velocity in the same range as for the open loop case.

The results show that the closed-loop control is efficient since each obtained functional value as well as average and instantaneous fields are close to the best results achieved by the open-loop control. As the figure 3 shows the averaged recirculation zones are remarquably reduced and concentrated in a small area behind the step using this closed-loop control.

These results are then compared to those obtained using intrusive sensor. More precisely, we now induce at the entry of the domain the velocity :

$$
u_{i n}(t)=1.0+M\left(u_{S_{i}}(t)-\overline{u_{S_{i}}}\right),
$$



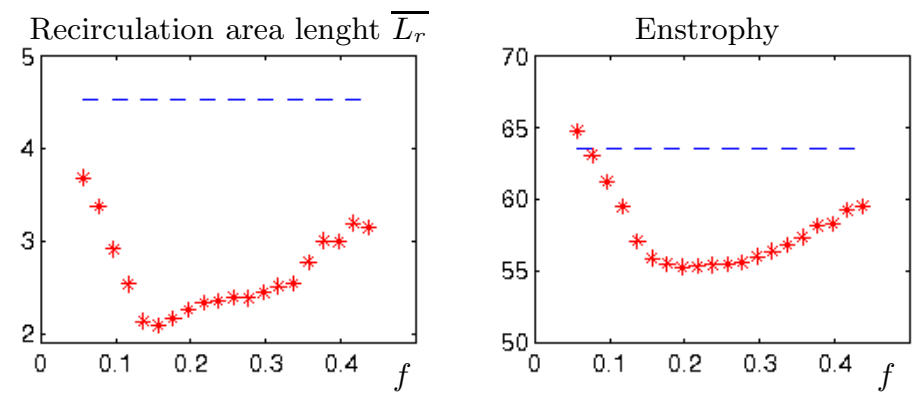

Near-wall recirculation area intensity

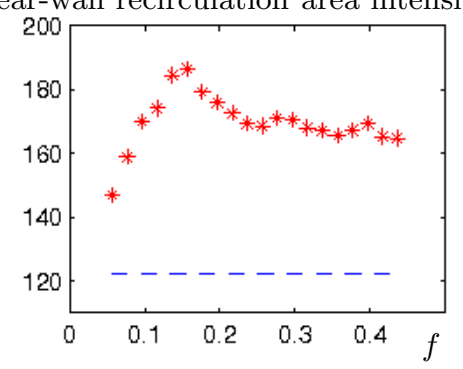

Fig. 2. Value of the functionals. Top left : Recirculation area lenght. Top right : Enstrophy. Bottom left : Near-wall recirculation intensity. In dashed-blue line : The value corresponding to uncontrolled flow.

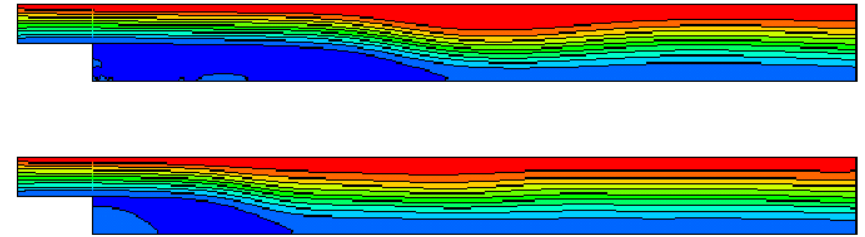

Fig. 3. Mean streamlines for uncontrolled (up) and non-intrusive $C L C$ (down) regimes.

where $u_{S_{i}}(t)$ is the horizontal velocity at a given point inside the flow, $\overline{u_{S_{i}}}$ its time-averaged value recorded during an uncontrolled simulation, and $M$ a parameter chosen to tune the amplitude of the signal (which has to be the same as in the non intrusive case). It shows the higher efficiency of the intrusive control compared to non intrusive one : the time-averaged value $L_{r}$ is respectively equal to 2.71 in the non intrusive case and 2.38 in the intrusive one ( 4.51 for the uncontrolled flow). Indeed, in the intrusive configuration the 
sensors are placed inside the flow that is less perturbated by the wall effects. This can be understood by looking at the value of $u_{i n}(t)$ in both cases (see Fig. 4).
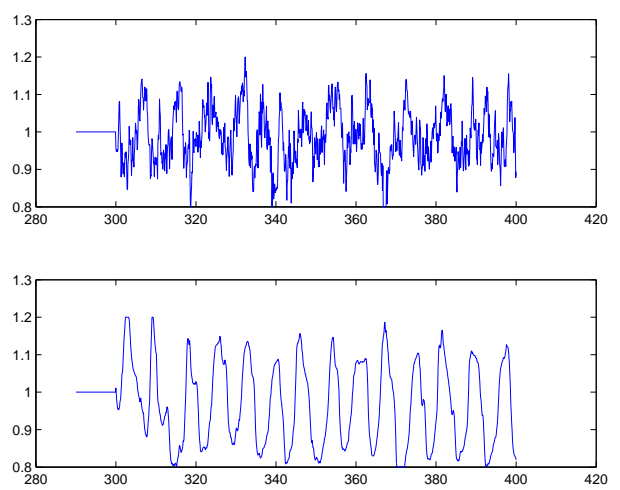

Fig. 4. Signal of $u_{i n}(t)$ for closed-loop control : with non intrusive sensor (top) and with intrusive sensor (bottom).

\subsection{Second control type}

In the second type of control, two small jets are implemented on the upper and lower parts of the vertical step (Figure 1, $\mathrm{Control}_{2}$ ). The main target of this approach is to influence directly the vortex shedding and the recirculation zone: the upper jet changes the shedding dynamics and the lower one perturbates and pushes away the recirculation zone. The actuators placed in the close vicinity of the bottom and the top of the step, blow or expell an horizontal flow inside the flow.

In this study, different possibilities as steady open-loop, active open-loop and active closed-loop actuators are applied to this configuration and their efficiency is verified. The inlet velocity is taken equal to unity; $u_{j u}$ and $u_{j b}$ correspond to the uniform jet velocities respectively on the top and the bottom of the step. Then, the oscillation, according to the control technique, is imposed to these velocities. Moreover, the computations are performed with both pulsed and synthetic jets. In the first case, in both devices the jet varies between 0 and $|2 A|$, with a negative jet in the upper side of the step and a positive one in the lower part. In the second, the jet varies between $-A$ and $A$ and there is no absolutely positive or negative device, even they never coincide to each other. 
For this kind of control, as the figure 5 shows, the best closed-loop results are achieved, taking $\left(u_{j u}, u_{j b}\right)=(-1.0,0.5)$ as the initial conditions and implementing the pulsed jets. A lot of detailed results are available in [4].

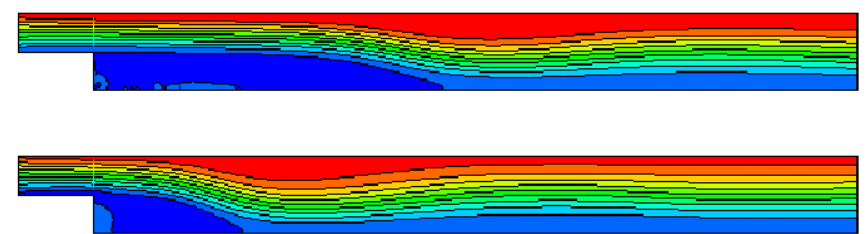

Fig. 5. Mean streamlines for uncontrolled (up) and $C L C$ with pulsed step jets (down) regimes.

\section{Conclusion}

In this work, both open and closed-loop control methods were applied to control a backward-facing step flow using a Vortex-In-Cell method. Two control configurations the first one correponding to a pulsing inlet flow and the second one to jets introduced into the lower and upper parts of the step were studied, . The first strategy showed that the natural fundamental frequency of the flow offers the characteristic value to tune the pulsing frequency. The control is therefore based on this value whatever the used devices. We also observed the efficiency of implementing the intrusive sensors. For the second strategy, the best control is achieved with a high velocity negative upper jet compared to a positive bottom jet. This result was also true for oscillating jets. It was verified that an automatic closed-loop approach can provide a control as efficient as the best open-loop control.

\section{References}

1. Cottet, G-H. and Koumoutsakos, P: Vortex methods, Cambridge Univ. Press. (2000)

2. Christiansen, J.P: Numerical Simulation of Hydrodynamics by the Method of Point Vortices. J. Comp. Phys., 13, 363-379 (1971)

3. Creusé, E. and Mortazavi, I.: Simulation of low Reynolds number flow control over a backward-facing step using pulsed inlet velocities. Applied Math. Research Express, 4, 133-152 (2004)

4. Creusé, E., Giovannini, A. and Mortazavi, I.: Vortex simulation of active control strategies for transitional backward-facing step flows. Computers and Fluids, (2008) in press.

5. Kaiktsis, L. and Monkewitz, P.: Global destabilization of flow over a backward-facing step. Phys. Fluids, 15, 3647-3658 (2003) 